\title{
2295. Multi-axis fatigue loading system of wind turbine blade and vibration coupling characteristics
}

\author{
Lei-an Zhang ${ }^{1}$, Li-ming Tao ${ }^{2}$, Xiu-ting Wei ${ }^{3}$, Xue-mei Huang ${ }^{4}$ \\ College of Mechanical Engineering, Shandong University of Technology, Shandong, China \\ ${ }^{1}$ Corresponding author \\ E-mail: 1ziaver@163.com, ${ }^{2} 1015639901 @ q q . c o m,{ }^{3}$ wxt@sdut.edu.cn, ${ }^{4}$ huangxuemei@sdut.edu.cn
}

Received 27 January 2016; received in revised form 18 August 2016; accepted 4 September 2016

DOI https://doi.org/10.21595/jve.2016.16879

Check for updates

\begin{abstract}
This paper presents a new method which focuses on the multi-axis fatigue loading mode for wind turbine blade and aims to shorten the fatigue loading cycle. The whole test scheme is design for the measurement of fatigue loading system. The two leading sources of fatigue loading system are asymmetric arrangement in the space. In addition, its vibration mathematical model is derived according to the Lagrange equation. The numerical simulation model is developed by means of Matlab Simulink. The vibration coupling characteristics including motor revolution speed, phase and amplitude of wind turbine blade is obtained. Moreover, the trajectory of wind turbine blade is obtained. Finally, a multi-axis fatigue loading platform for small wind turbine blade is built for the proposed study. The on-site test showed that if the revolution speeds of the two loading sources is the same as the natural frequency of wind turbine blade, the revolution speed, the phase angle of motor and the blade trajectory were relative changed smoothly. Thus, the amplitude of blade is state and the largest. Otherwise when the revolution speed of motor is different with the natural frequency of blade, the revolution speeds and phase angle of the two loading sources fluctuated largely. The above conclusion provided the theoretical basis for the subsequent decoupling control algorithm of multi-axis fatigue loading test.
\end{abstract}

Keywords: wind turbine blade, multi-axis loading mode, mathematical model, numerical simulation, fatigue loading test.

\section{Introduction}

Among all renewable energies, the wind energy has shown very broad applications. The main component of wind turbine is wind turbine blade. Its main failure mode is fatigue damage because of the alternate loading. Thus, the fatigue loading test is an effective method to check the anti-fatigue property for wind turbine blade [1-3]. Generally, fatigue loading test apply to the single-axis excitation mode at home and abroad, and there are two problems in this loading mode. Firstly, the flap wise and edgewise direction of blade needs to do fatigue loading test respectively. But the measurement is lengthy and time consuming. Secondly, according to the statistical report of National Renewable Energy Laboratory, the phase angle between the two loading direction of blade is approximately present Gauss distribution. Moreover, the probability of phase angle is 72 degrees, because of the variation of the wind direction (shown in Fig. 1). The actual force of blade should be the synthetic force of two direction loading (shown in Fig. 2). So the single-axis loading mode is inappropriate in real application. This could lead to the inaccuracy of the test result. Based on the above discussion, this paper proposed a new multi-axis fatigue loading method driven by electric mass in order to get the accurate measurement results of wind turbine blade. In this loading method, the space structure of two loading sources is in asymmetric condition. An electromechanical coupling system has been form between them and the blade. Thus, the proposed vibration characteristics of multi-axis loading system has the good theoretical significance for the subsequent fatigue test. There were a lot of researches with the two loading sources vibration field already have done. Massicotte et al. obtained the theoretical conditions for the vibration self-synchronization of the loading system according to the mathematical model of vibration coupling and at the same time analyzed the variation laws by using simulation model [57]. As for the stability of multi-excitation vibration, De-gang Wang analyzed the dynamic 
vibration characteristics of the multi-excitation system quantitatively through establishing its accurate dynamic model [8]. Tian-xia Zhang applied the nonlinear method to establish the model of the loading system driven by the eccentric mass. Its mathematical description of structural parameters was given, and the variable range of vibration parameters was determined [9]. The above results indicated that vibration coupling between the two loading sources is inherent. The research method also provided a good reference value for the vibration characteristics of wind turbine blade with multi-axis loading system.

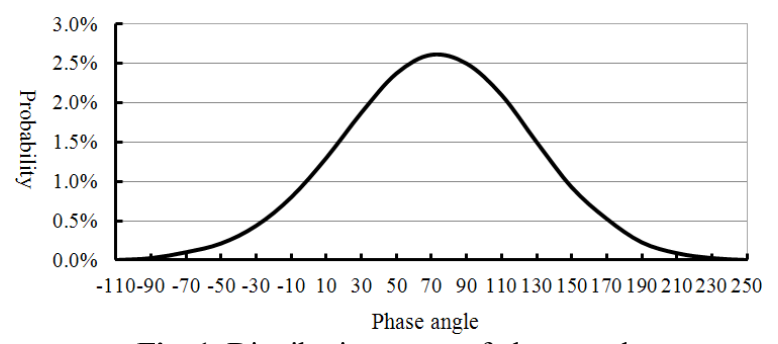

Fig. 1. Distribution curve of phase angle

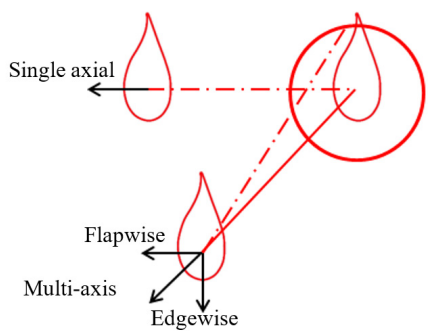

Fig. 2. Actual loading direction of wind turbine blade

\section{Design of fatigue loading system}

\subsection{Multi-axis loading scheme}

The multi-axis fatigue loading system (Fig. 3) mainly included loading base, wind turbine blade and two fatigue loading sources. The two loading sources were connected with the wind turbine blade by wood saddle. The loading base was fixed on the ground, and the blade root was fixed on the loading base through a high strength bolts. Loading source was composed of AC motor, a reducer, a connecting clamp and a control cabinet. Two laser range devices were used to measure the amplitude of two loading direction, and the two pulse sensor were used to measure the revolution speed of loading source.

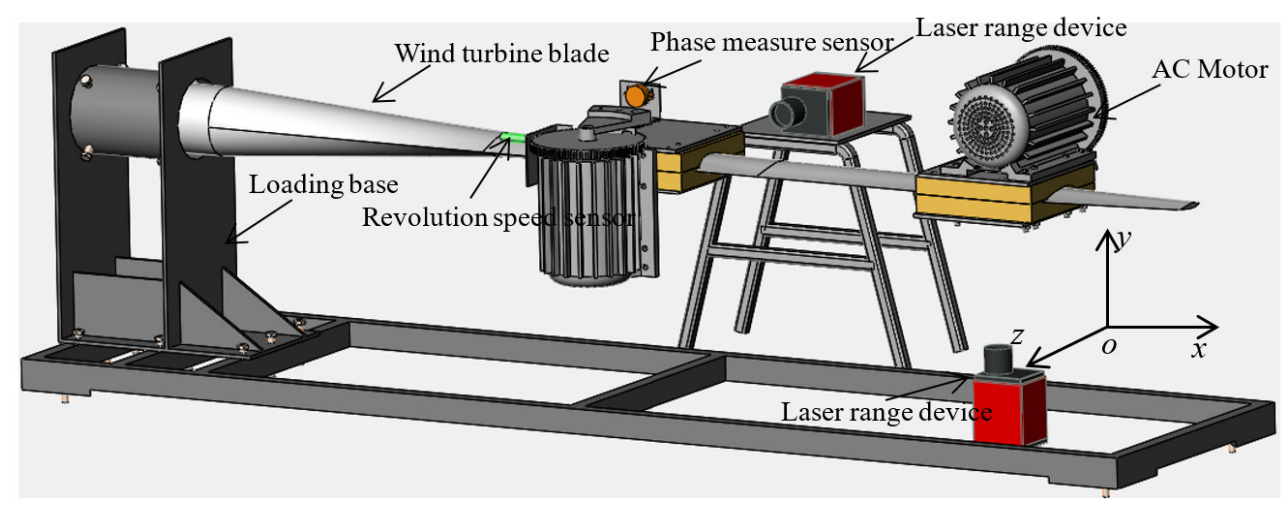

Fig. 3. Multi-axis fatigue loading scheme of wind turbine blade

\subsection{Measurement of speed difference, phase difference and amplitude}

High counting sensor which adopting advanced detection technology could identify every teeth of gear and convert it into a square wave output. Output shaft of each loading source installed with a measuring gear and two sensors measuring the revolution speed and phase angle, as shown in Fig. 4. The speed measuring gear had 180 teeth and each of which represented two angles. There was a zero mark on the speed measuring gear. During the measuring, each gear speed sensor 
produced a pulse when a tooth of gear was detected, so the driving speeds and phases of the unbalanced shafts could be obtained. A proximity switch is installed below every unbalanced shaft to realize the reset of phase.

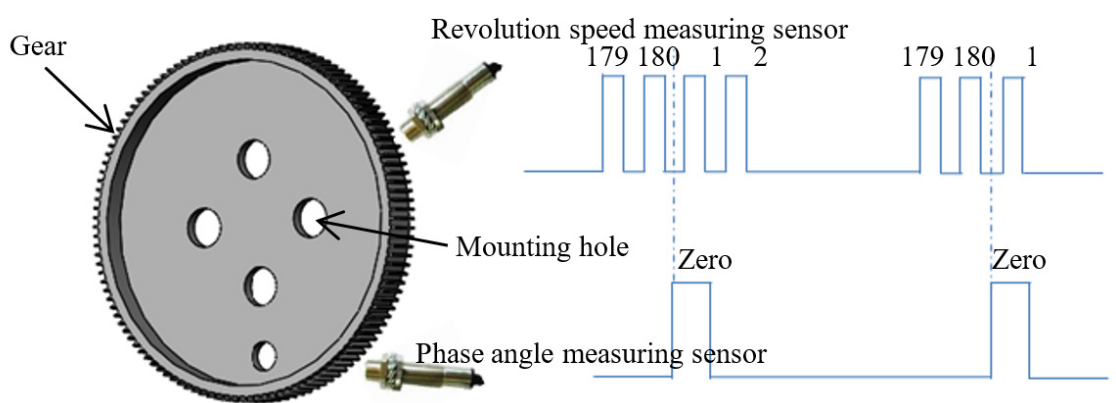

Fig. 4. Speed difference and phase difference detection scheme

The centrifugal force generated by each eccentric mass was defined as the center of gravity line (denoted by $M_{1}$ and $M_{2}$ ). The two center line of gravity could be maintained at a constant angle, which indicated that the two loading source vibrated synchronization. Take the two loading sources for example, the revolution speed difference $\Delta v(t)(\mathrm{rad} / \mathrm{s})$ and phase angle difference $\Delta \varphi(t)(\mathrm{rad})$ could be expressed as:

$$
\begin{aligned}
& \Delta v(t)=v_{1}(t)-v_{2}(t)=\frac{d M_{1}(t)-d M_{2}(t)}{d t}, \\
& \Delta M(t)=\Delta M(0)+\int \Delta v(t) t d t m \\
& \Delta \varphi(t)= \begin{cases}\Delta M(t)-2 \pi, & \Delta M(t) \geq 2 \pi, \\
\Delta M(t)+2 \pi, & \Delta M(t) \leq-2 \pi, \\
\Delta M(t), & -2 \pi<\Delta M(t)<2 \pi,\end{cases}
\end{aligned}
$$

where: $v_{1}(t), v_{2}(t)$ - revolution speed of eccentric mass; $M_{1}(t), M_{2}(t)$ - the position of eccentric mass; $\Delta \varphi(t)$ - phase angle difference; $\Delta M(0)$ - initial position.

The differential detection of revolution speed and phase angle could be realized according to Eqs. (1)-(3).

Non-contact laser measurement was used to avoid damage for the measuring equipment because of the blade vibration. Measurement equipment was laser range device. The laser range mode was set a one-way transmission mode and communication format was set to: 19200.EVEN.8.1. Data transmission cycle was $T=30 \mathrm{~ms}$. The blade amplitude was represented

\begin{tabular}{|c|c|c|c|c|c|c|c|}
\hline 7 bit & 6bit & 5bit & 4bit & 3 bit & 2 bit & 1bit & Obit \\
\hline \multicolumn{6}{|c|}{ Data (L) } & 0 & 0 \\
\hline 7 bit & 6bit & 5 bit & 4bit & 3 bit & 2bit & 1bit & Obit \\
\hline \multicolumn{6}{|c|}{ Data (M) } & 0 & 1 \\
\hline 7 bit & 6 bit & 5 bit & 4bit & 3 bit & 2 bit & 1bit & Obit \\
\hline$x$ & $x$ & \multicolumn{4}{|c|}{ Data $(\mathrm{H})$} & 1 & 0 \\
\hline
\end{tabular}
by three bytes. The commutation protocol was shown in Table 1 .

Table 1. Laser ranging protocol

According to the communication protocol of Table 1, the formula of blade amplitude was calculated as follows: Amplitude $=(\mathrm{L} \gg 2)+(\mathrm{M} \gg 2) * 64+((\mathrm{H} \gg 2) \& 0 \times 0 \mathrm{~F}) * 4096$; 


\section{Electromechanical coupling dynamic model}

\subsection{Mathematical model}

According to the multi-axis loading scheme of wind turbine as shown in Fig. 3, some assumptions were made as the following:

(1) The blade was flexible, and the loading source was rigidly connected to the blade.

(2) When the blade vibrates, damping force and elastic force were proportional to the velocity and displacement of the blade respectively.

(3) xoy plane was in the blade oriented direction, and xoz plane was in the blade tangential direction.

Three coordinate systems and five degree freedoms were defined: translatory motion along the $x$ axis, $y$ axis, $z$ axis and rotation around the $x$ axis, $z$ axis respectively. The proposed Dynamic model as shown in Fig. 5.

As shown in Fig. 5, oxyz was the absolute coordinate system, $o^{\prime} x^{\prime} y^{\prime} z^{\prime}$ and $o^{\prime \prime} x^{\prime \prime} y^{\prime \prime} z^{\prime \prime}$ were the moving coordinate systems, $o^{\prime}\left(o^{\prime \prime}\right)$ was motion synthesis centroid of the system; $o^{\prime \prime \prime}$ was the centroid of the whole vibration system (including the blade and the two loading sources). In addition, $M$ was the equivalent weight of the blade; $o_{1}$ and $o_{2}$ were the rotary center of two eccentric mass respectively. And, $l_{1}$ and $l_{1}^{\prime}$ were the projection of $o o_{1}$ in the plane $x o y$ and $y o z$ respectively; $l_{2}$ and $l_{2}^{\prime}$ were the projection of $o o_{2}$ in the plane $x o y$ and yoz respectively. The angle between $l_{1}, l_{2}$ were $\beta_{1}$ and $\beta_{2}$ and the positive direction of $x$-axis respectively. $\beta_{1}^{\prime}$ was the angle between $l_{1}^{\prime}$ and the negative direction of $z$-axis and $\beta_{2}^{\prime}$ was the angle between $l_{2}^{\prime}$ and the positive direction of $z$-axis; $m_{i}(i=1,2)$ was the equivalent weight of the eccentric mass; $r_{i}$ $(i=1,2)$ was the equivalent eccentricity of the eccentric mass; $\theta_{1}$ and $\theta_{2}$ were the rotation angle of the eccentric mass respectively, and $\omega_{i}=d \theta_{i} / d t(i=1,2) ; \varphi$ was the rise angle of connecting $\operatorname{rod} m_{2}$.

The kinetic energy $T$ of the proposed system was expressed as:

$$
\begin{aligned}
T= & \frac{1}{2} M\left[\left(\dot{x}+\dot{\varphi}_{1} l_{0} \sin \beta_{0}\right)^{2}+\left(\dot{y}-\dot{\varphi}_{1} l_{0} \cos \beta_{0}-\dot{\varphi}_{2} l_{0}^{\prime} \sin \beta_{0}^{\prime}\right)^{2}+\left(\dot{z}-\dot{\varphi}_{2} l_{0}^{\prime} \cos \beta_{0}^{\prime}\right)^{2}\right] \\
& +\frac{1}{2} \sum_{i=1}^{2} J_{i} \dot{\varphi}_{i}^{2}+\frac{1}{2} m_{1}\left[\left(\dot{x}-\dot{\varphi}_{1} l_{1} \sin \beta_{1}+\dot{\theta}_{1} r_{1} \cos \theta_{1}\right)^{2}+\left(\dot{y}+\dot{\varphi}_{1} l_{1} \cos \beta_{1}+\dot{\varphi}_{2} l_{1}^{\prime} \cos \beta_{1}^{\prime}\right.\right. \\
& \left.\left.+\dot{\theta}_{1} r_{1} \sin \theta_{1}\right)^{2}+\left(\dot{z}+\dot{\varphi}_{2} l_{1}^{\prime} \sin \beta_{1}^{\prime}\right)^{2}\right]+\frac{1}{2} m_{2}\left[\left(\dot{x}+\dot{\varphi}_{1} l_{2} \sin \beta_{2}-\dot{\theta}_{2} r_{2} \sin \theta_{2}\right)^{2}\right. \\
& \left.+\left(\dot{y}+\dot{\varphi}_{1} l_{2} \cos \beta_{2}+\dot{\varphi}_{2} l_{2}^{\prime} \cos \beta_{2}^{\prime}\right)^{2}+\left(\dot{z}+\dot{\varphi}_{2} l_{2}^{\prime} \sin \beta_{2}^{\prime}-\dot{\theta}_{2} r_{2} \cos \theta_{2}\right)^{2}\right]+\frac{1}{2} \sum_{i=1}^{2} J_{i}^{\prime} \dot{\theta}_{i}^{2}
\end{aligned}
$$

where, $J_{i}(i=1,2)$ is the equivalent moment of inertia of the whole system; $J_{i}^{\prime}(i=1,2)$ is the equivalent moment of inertia of the eccentric mass.

The potential energy $V$ of the system was expressed as:

$V=\frac{1}{2} k(x) x^{2}+\frac{1}{2} k(y) y^{2}+\frac{1}{2} k(z) z^{2}+\frac{1}{2} k_{\varphi 1} \varphi_{1}{ }^{2}+\frac{1}{2} k_{\varphi_{2}} \varphi_{2}{ }^{2}$,

where, $k(x), k(y), k(z), k_{\varphi 1}, k_{\varphi 2}$ are the equivalent stiffness of these directions.

The kinetic energy $T$ and potential energy satisfied the following equation according to the Lagrange equation:

$$
\frac{d}{d t} \frac{\partial T}{\partial \dot{q}_{i}}-\frac{\partial T}{\partial q_{i}}+\frac{\partial V}{\partial q_{i}}=Q_{i}, \quad(i=1,2,3 \ldots) .
$$


External force of the system $Q$ was expressed as:

$$
Q_{i}=\left[\begin{array}{c}
-c_{x} \dot{x} \\
-c_{y} \dot{y} \\
-c_{z} \dot{z} \\
-c_{\varphi 1} \dot{\varphi}_{1} \\
-c_{\varphi 2} \dot{\varphi}_{2} \\
T_{m 1}-T_{f 1}-c_{1} \dot{\theta}_{1} \\
T_{m 2}-T_{f 2}-c_{2} \dot{\theta}_{2}
\end{array}\right],
$$

where, $c_{x}, c_{y}, c_{z}, c_{\varphi i}(i=1,2)$ are the equivalent damping of vibration direction; $c_{i}(i=1,2)$ is the equivalent damping of motor output shaft and, $T_{m i}, T_{f i}(i=1,2)$ are the electromagnetic torque and load torque of motor.

$x, y, z, \varphi_{i}, \theta_{i}(i=1,2)$ were regarded as generalized coordinates. Then include the kinetic energy, potential energy and the energy dissipation function of the whole system into the Lagrange equation, and the dynamic equation of the multi-axis fatigue loading system was established as follows:

$$
\left\{\begin{array}{l}
\left(M+m_{1}+m_{2}\right) \ddot{x}+m_{1} r_{1}\left(\ddot{\theta}_{1} \cos \theta_{1}-\dot{\theta}_{1}^{2} \sin \theta_{1}\right)-m_{2} r_{2}\left(\ddot{\theta}_{2} \sin \theta_{2}+\dot{\theta}_{2}^{2} \cos \theta_{2}\right) \\
\quad+k(x) x+c_{x} \dot{x}=0, \\
\left(M+m_{1}+m_{2}\right) \ddot{y}+m_{1} r_{1}\left(\ddot{\theta}_{1} \sin \theta_{1}+\dot{\theta}_{1}^{2} \cos \theta_{1}\right)+k(y) y+c_{y} \dot{y}=0 \\
\left(M+m_{1}+m_{2}\right) \ddot{z}-m_{2} r_{2}\left(\ddot{\theta}_{2} \cos \theta_{2}-\dot{\theta}_{2}^{2} \sin \theta_{2}\right)+k(z) z+c_{z} \dot{z}=0 \\
\left(M l_{0}^{2}+m_{1} l_{1}^{2}+m_{2} l_{2}^{2}+J_{1}\right) \ddot{\varphi}_{1}+\left(M l_{0} l_{0}^{\prime} \sin \beta_{0} \sin \beta_{0}^{\prime}+m_{1} l_{1} l_{1}^{\prime} \cos \beta_{1} \cos \beta_{1}^{\prime}\right. \\
\left.\quad+m_{2} l_{2} l_{2}^{\prime} \cos \beta_{2} \cos \beta_{2}^{\prime}\right) \ddot{\varphi}_{2}+k\left(\varphi_{1}\right) \varphi_{1}+c_{\varphi 1} \dot{\varphi}_{1}=m_{1} r_{1} l_{1} \sin \beta_{1}\left(\ddot{\theta}_{1} \cos \theta_{1}-\dot{\theta}_{1}^{2} \sin \theta_{1}\right) \\
\quad-m_{1} r_{1} l_{1} \cos \beta_{1}\left(\ddot{\theta}_{1} \sin \theta_{1}+\dot{\theta}_{1}^{2} \cos \theta_{1}\right)+m_{2} r_{2} l_{2} \sin \beta_{2}\left(\ddot{\theta}_{2} \sin \theta_{2}+\dot{\theta}_{2}^{2} \cos \theta_{2}\right) \\
\left(M l_{0}^{2}+m_{1} l_{1}^{\prime 2}+m_{2} l_{2}^{\prime 2}+J_{2}\right) \ddot{\varphi}_{2}+\left(M l_{0} l_{0}^{\prime} \cos \beta_{0} \sin \beta_{0}^{\prime}+m_{1} l_{1} l_{1}^{\prime} \cos \beta_{1} \cos \beta_{1}^{\prime}\right. \\
\left.\quad+m_{2} l_{2} l_{2}^{\prime} \cos \beta_{2} \cos \beta_{2}^{\prime}\right) \ddot{\varphi}_{1}+k\left(\varphi_{2}\right) \varphi_{2}+c_{\varphi_{2}} \dot{\varphi}_{2}=m_{2} r_{2} l_{2}^{\prime} \sin \beta_{2}^{\prime}\left(\ddot{\theta}_{2} \cos \theta_{2}-\dot{\theta}_{2}^{2} \sin \theta_{2}\right) \\
\quad-m_{1} r_{1} l_{1}^{\prime} \cos \beta_{1}^{\prime}\left(\ddot{\theta}_{1} \sin \theta_{1}+\dot{\theta}_{1}^{2} \cos \theta_{1}\right) m_{1} r_{1}\left[\left(\ddot{x}_{1}-\ddot{\varphi}_{1} l_{1} \sin \beta_{1}\right) \cos \theta_{1}\right. \\
\left.\quad+\left(\ddot{y}+\ddot{\varphi}_{1} l_{1} \cos \beta_{1}+\ddot{\varphi}_{2} l_{1}^{\prime} \cos \beta_{1}^{\prime}\right) \sin \theta_{1}+\ddot{\theta}_{1} r_{1}\right]+J^{\prime} \ddot{\theta}_{1}=T_{m 1}-T_{f 1}-c_{1} \dot{\theta}_{1} m_{2} r_{2}\left[\ddot{\theta}_{2} r_{2}\right. \\
\left.\quad-\left(\ddot{x}+\ddot{\varphi}_{1} l_{2} \sin \beta_{2}\right) \sin \theta_{2}-\left(\ddot{z}+\ddot{\varphi}_{2} l_{2}^{\prime} \sin \beta_{2}^{\prime}\right) \cos \theta_{2}\right]+J^{\prime} \ddot{\theta}_{2}=T_{m 2}-T_{f 2}-c_{2} \dot{\theta}_{2} .
\end{array}\right.
$$

\subsection{Mathematical model of AC motor}

Three-phase asynchronous motor provided power for the loading sources, and its state equation under the two-phase synchronous rotating coordinate system could be represented as Eq. (9) [10-11]:

$$
\begin{aligned}
{\left[\begin{array}{l}
\dot{I}_{d s} \\
\dot{I}_{q s} \\
\dot{I}_{d r} \\
\dot{I}_{q r}
\end{array}\right] } & {\left[\begin{array}{cccc}
L_{s} R_{s} & \omega_{1}\left(L_{s} L_{r}-L_{m}^{2}\right)+\omega_{r} L_{m}^{2} & -L_{m} R_{r} & -\omega_{r} L_{m} L_{r} \\
\omega_{1}\left(L_{s} L_{r}-L_{m}^{2}\right)+\omega_{r} L_{m}^{2} & L_{r} R_{s} & L_{r} L_{m} \omega_{r} & -L_{m} R_{r} \\
-L_{m} R_{s} & L_{s} L_{m} \omega_{r} & L_{s} R_{r} & \omega_{1}\left(L_{s} L_{r}-L_{m}^{2}\right) \\
-L_{s} L_{m} \omega_{r} & -L_{m} R_{s} & \omega_{1}\left(L_{s} L_{r}-L_{m}^{2}\right) & R_{r} L_{s}
\end{array}\right] } \\
& \times \frac{1}{L_{m}^{2}-L_{s} L_{r}}\left[\begin{array}{c}
I_{d s} \\
I_{q s} \\
I_{d r} \\
I_{q r}
\end{array}\right]+\left[\begin{array}{cccc}
L_{r} & 0 & -L_{m} & 0 \\
0 & -L_{r} & 0 & L_{m} \\
L_{m} & 0 & -L_{s} & 0 \\
0 & L_{m} & 0 & -L_{s}
\end{array}\right]\left[\begin{array}{l}
U_{d s} \\
U_{q s} \\
U_{d r} \\
U_{q r}
\end{array}\right] \dot{\omega}_{r}
\end{aligned}
$$




$$
=\frac{n_{p}^{2}}{J_{m}} L_{m}\left(I_{q 1} I_{d 2}-I_{q 2} I_{d 1}\right)-\frac{D}{J_{m}} \omega_{r}-\frac{n_{p}}{J_{m}} T_{L}
$$

where, $U_{d s}, U_{q s}, U_{d r}, U_{q r}$ - stator terminal voltage, rotor terminal voltage under two-phase synchronous rotating coordinate system; $I_{d s}, I_{q s}, I_{d r}, I_{q r}$ - stator terminal current, rotor terminal current under two-phase synchronous rotating coordinate system; $R_{s}, R_{r}, L_{s}, L_{r}, L_{m}$-stator, rotor resistance, self- inductance and mutual inductance; $\omega_{1}, \omega_{r}$ - synchronous rotating angular velocity and the angular velocity of rotor; $D, T_{L}, n_{p}, J_{m}$ - coefficient of resistance moment, the load torque of the motor, pole pairs and inertia moment; $i_{d 1}, i_{d 2}$ - component of stator current under $d$ shafting; $I_{q 1}, I_{q 2}$ - Component of stator current under $q$ shafting.

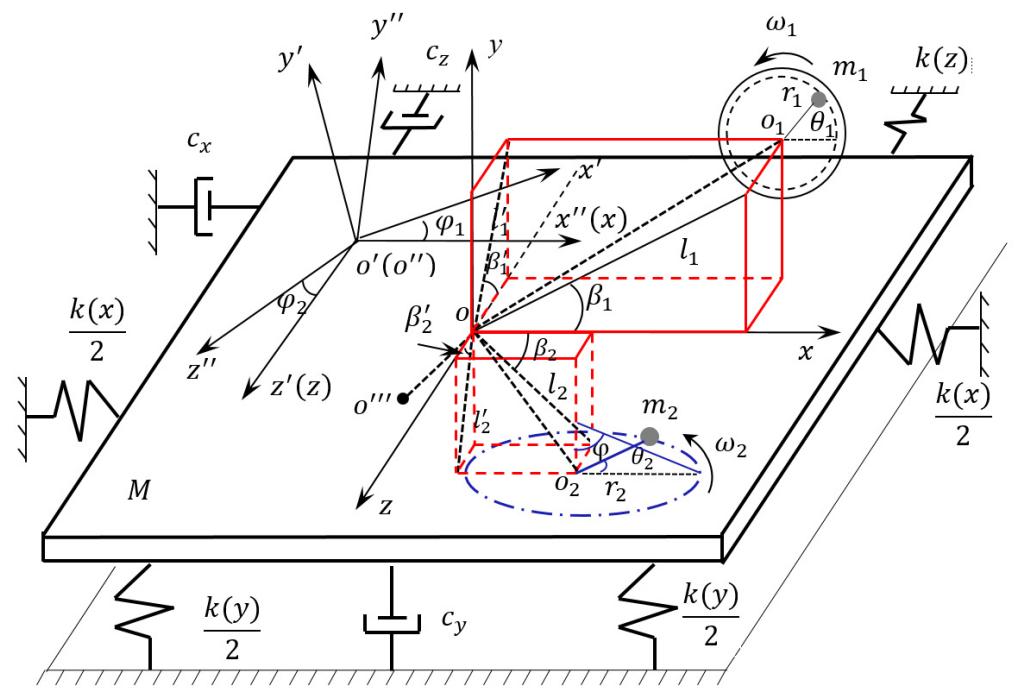

Fig. 5. Dynamic model of multi-axial loading mode

\subsection{Simulation model}

It was difficult to get the exact solution of mathematical model from above equations because of the multi variable electromechanical coupling effect. In order to get the vibration characteristics of multi-axis fatigue loading system visually, simulation model based on Matlab/Simulink software was established according to mathematical model. It was showed in Fig. 6. Motor A and Motor B were two loading sources. Every simulation model of AC motor included voltage model, electromagnetic torque model, motion model and flux linkage torque model.

\subsection{Analysis of simulation test}

The simulation model parameters were set as: $M=25 \mathrm{~kg}, m_{1}=m_{2}=2 \mathrm{~kg}, r_{1}=0.15 \mathrm{~m}$, $r_{2}=0.15 \mathrm{~m}, l_{1}=0.05 \mathrm{~m}, l_{2}=0.25 \mathrm{~m}, l_{1}^{\prime}=0.06 \mathrm{~m}, l_{2}^{\prime}=0.16 \mathrm{~m}, \beta_{1}=\beta_{2}=14^{\circ}, \beta_{1}^{\prime}=42^{\circ}$, $\beta_{2}^{\prime}=48^{\circ}$. The initial phase angle of two loading sources was zero. The initial setting revolution speeds of the loading source were $n_{1}=50 \mathrm{r} / \mathrm{min}$ and $n_{2}=55 \mathrm{r} / \mathrm{min}$ in flapwise direction. The initial setting revolution speeds of the loading source were $n_{3}=70 \mathrm{r} / \mathrm{min}$ and $n_{4}=75 \mathrm{r} / \mathrm{min}$ in edgewise direction. $n_{1}=50 \mathrm{r} / \mathrm{min}$ and $n_{3}=70 \mathrm{r} / \mathrm{min}$ were the same as the nature frequency of blade in flapwise and edgewise direction respectively. The revolution speed and phase angle variable simulation results of the loading source were shown in Fig. 7 and Fig. 8. The amplitude of wind turbine blade and the overall trajectories of the flapwise direction were shown in Fig. 9. 


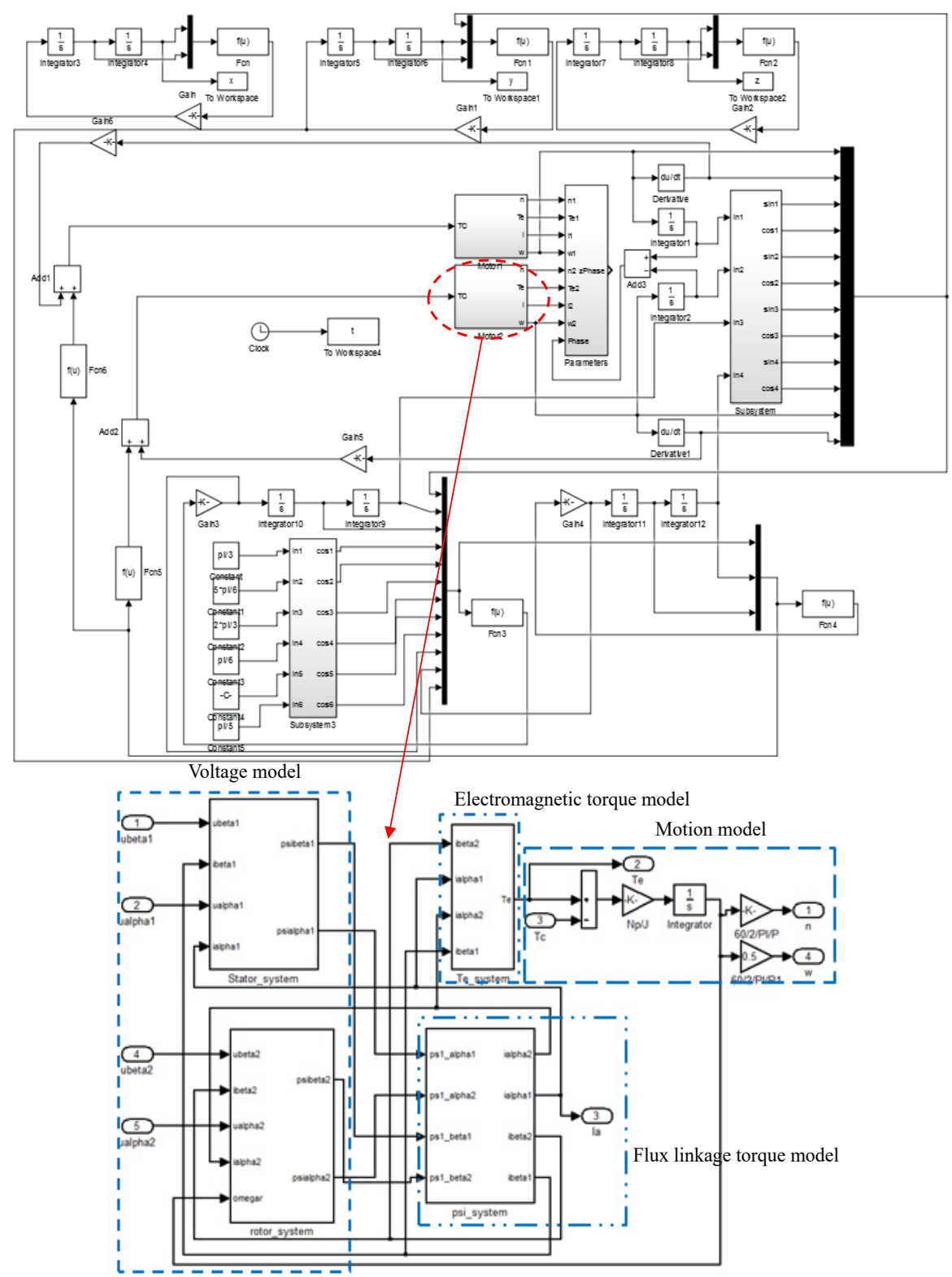

Fig. 6. The simulation model of multi-axis excitation system

The above simulation results showed that when the excitation frequency of the two loading source was same to the natural frequency of the blade vibration direction, the revolution speed of two loading sources of loading tend to be stable as shown in Fig. 7(a) and Fig. 8(a). The phase angle variation in two directions was relatively stable, as shown in Fig. 7(c) and Fig. 8(c). The vibration trajectory, not only the flapwise direction but also two directions, could get a stable large amplitude relatively, as shown in Fig. 9(a) and Fig. 9(c). When the excitation frequency of two loading source were differ to a large extent with the nature frequency of blade respectively, the revolution speeds of two loading sources were difficult to achieve vibration synchronous due to the inherent electromechanical coupling effect as shown in Fig. 7(b) and Fig. 8(b). The phase angle variation and the overall trajectory of wind turbine were disorder as shown in Fig. 7(c), Fig. 8(c), Fig. 9(b) and Fig. 9(d). 

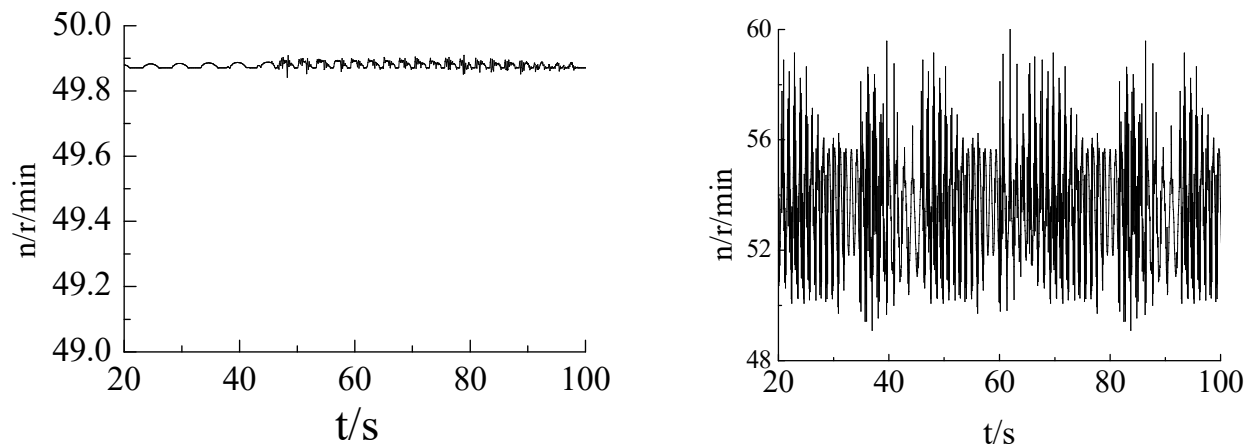

a) $n_{1}=50 \mathrm{r} / \mathrm{min}$, revolution speed of loading source b) $n_{2}=55 \mathrm{r} / \mathrm{min}$, revolution speed of loading source
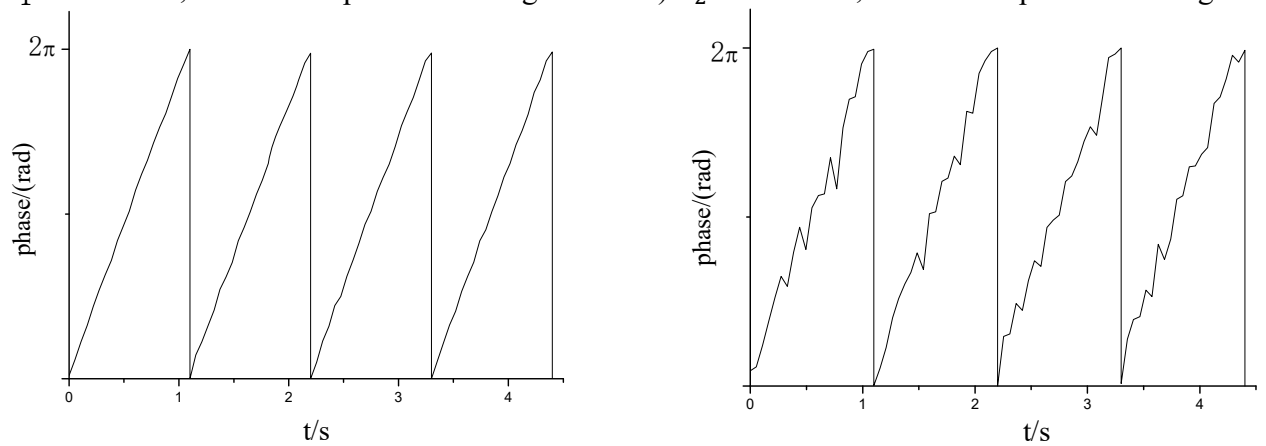

c) $n_{1}=50 \mathrm{r} / \mathrm{min}$, phase angle of loading source

d) $n_{2}=55 \mathrm{r} / \mathrm{min}$, phase angle of loading source

Fig. 7. Revolution speed and phase angle simulation of loading source in flapwise direction
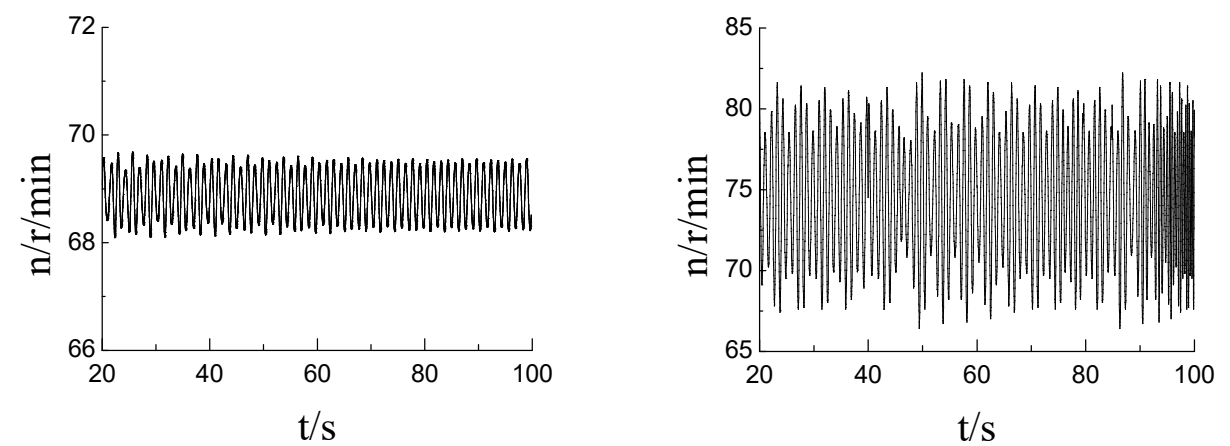

a) $n_{3}=70 \mathrm{r} / \mathrm{min}$, revolution speed of loading source b) $n_{4}=75 \mathrm{r} / \mathrm{min}$, revolution speed of loading source

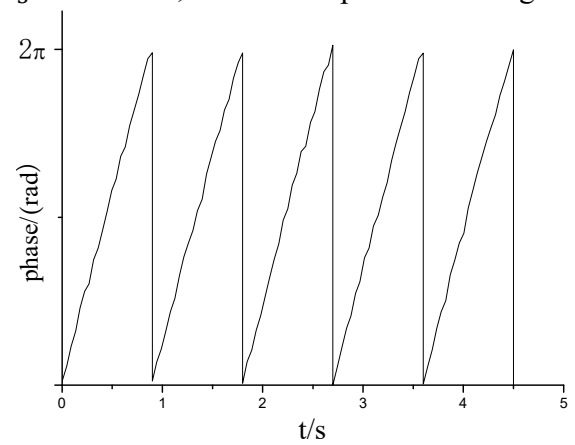

c) $n_{3}=70 \mathrm{r} / \mathrm{min}$, phase angle of loading source

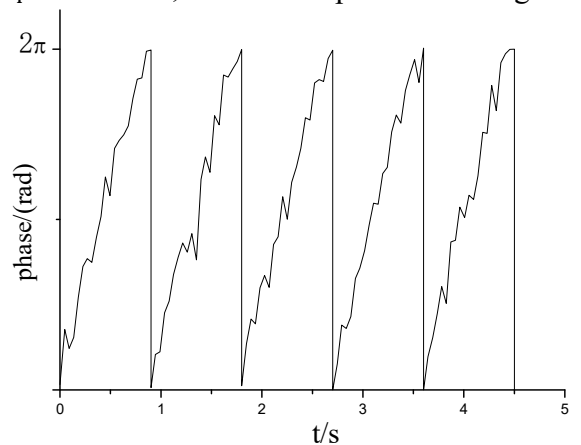

d) $n_{4}=75 \mathrm{r} / \mathrm{min}$, phase angle of loading source

Fig. 8. Revolution speed and phase angle simulation of loading source in edgewise direction 


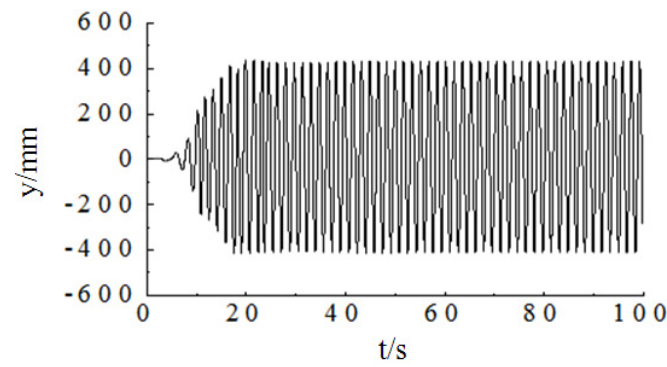

a) $n_{1}=50 \mathrm{r} / \mathrm{min}$, amplitude simulation curve of flapwise direction

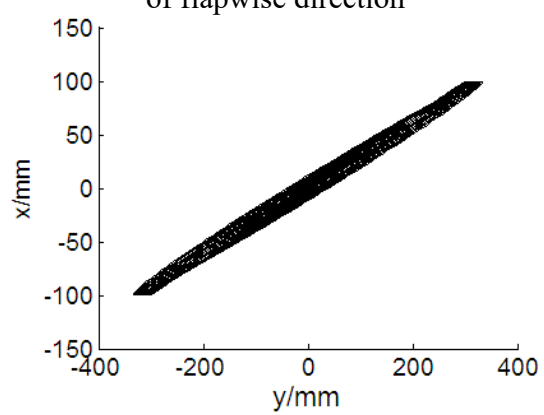

c) $n_{1}=50 \mathrm{r} / \mathrm{min}, n_{3}=70 \mathrm{r} / \mathrm{min}$, vibration trajectory of blade

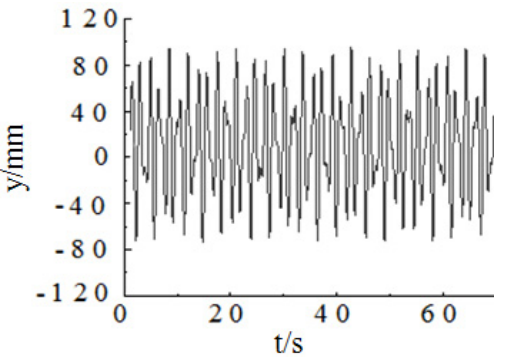

b) $n_{2}=55 \mathrm{r} / \mathrm{min}$, amplitude simulation curve of flapwise direction

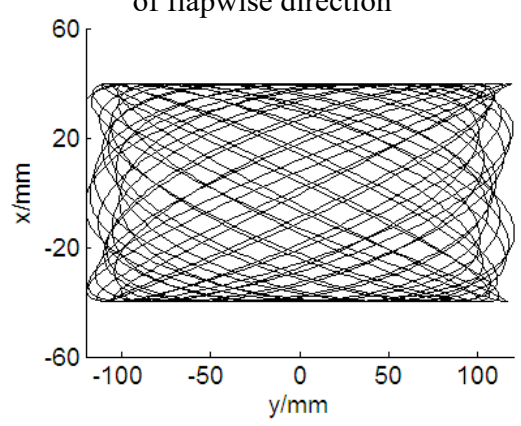

d) $n_{2}=55 \mathrm{r} / \mathrm{min}, n_{4}=75 \mathrm{r} / \mathrm{min}$, vibration trajectory of blade

Fig. 9. Vibration trajectory simulation of wind turbine blade

\section{Test verification}

\subsection{Experiment platform}

In order to verify the theoretical analysis and the simulation results, a multi-axis fatigue loading test system of small wind turbine blade was set up. The HMI monitoring interface based on Labview software was developed as shown in Fig. 10.

The whole control system used the distributed network bus mode, which was shown in Fig. 11. Two slave controllers controlling the frequency converter adjusted the revolution speed of every loading source. Laser range devices, measuring the amplitude of the blade through the RS485 bus, transmitted data to the controller. The main controller and the slave controllers exchanged the data through the CAN bus. The main controller and the monitoring interface also used the RS485 bus to realize the data exchange. All useful information was displayed and saved on the monitor interface.

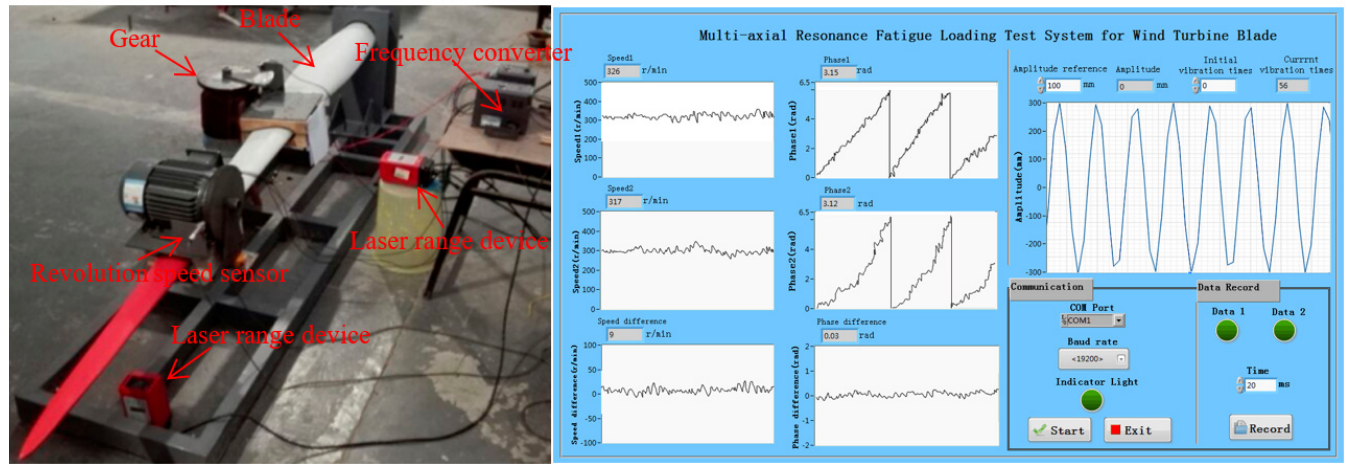

Fig. 10. Test platform and interface monitor 


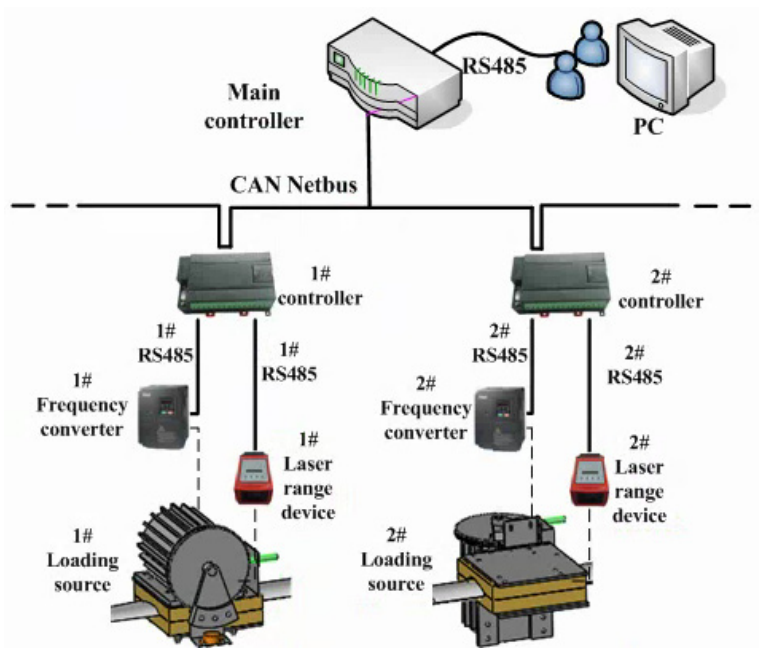

Fig. 11. Distributed network control architecture

Infinon XC164CS MCU was used for control the system hardware. The software in Keil3.0 compiler environment used $\mathrm{C}$ language to complete. The measure device for revolution speed was used the high-speed counting sensor and gear. Test parameters were shown in Table 2.

Table 2. Test parameters

\begin{tabular}{|c|c|}
\hline Parameters & Value \\
\hline Blade length $(\mathrm{m})$ & 2.3 \\
\hline Blade weight $(\mathrm{kg})$ & 25 \\
1st frequency in flapwise (Hz) & 0.86 \\
1st frequency in edgewise $(\mathrm{Hz})$ & 1.15 \\
\hline Eccentric mass $(\mathrm{kg})$ & 2 \\
\hline Eccentric length $(\mathrm{m})$ & 0.15 \\
\hline Motor power $(\mathrm{kW})$ & 0.75 \\
Frequency converter & ABB \\
Laser range device & ADSL-30 \\
Sensor type & CORON-CTS \\
\hline Test temperature $\left({ }^{\circ} \mathrm{C}\right)$ & 23 \\
\hline Humidity $(\mathrm{RH})$ & $36 \%$ \\
\hline
\end{tabular}

\subsection{Test result and analysis}

When the revolution speed of the loading source was respective setting with $50 \mathrm{r} / \mathrm{min}$ and $55 \mathrm{r} / \mathrm{min}$ in flapwise direction, the revolution speed and phase angle variation of loading source were measured as shown in Fig. 12. According to the test results, when the setting revolution speed was same to the natural frequency of blade $(n=50 \mathrm{r} / \mathrm{min})$, revolution speed of loading source tends to be stable, and the phase angle varied smoothly. When the setting revolution speed was different with the natural frequency of blade $(n=55 \mathrm{r} / \mathrm{min})$, due to the coupling effect between them, the revolution speed and phase angle variation of loading source fluctuated significantly. Above test measure results were basically match with the simulation results.

When the revolution speed of the loading source was also respective setting $70 \mathrm{r} / \mathrm{min}$ and $75 \mathrm{r} / \mathrm{min}$ in edgewise direction, the revolution speed of loading source was measured as shown in Fig. 13. According to the test results, when the setting revolution speed was same with the natural frequency of blade ( $n=70 \mathrm{r} / \mathrm{min}$ ), revolution speed of loading source tends to be stable and the phase angle varied smoothly. When the setting revolution speed was different with the natural frequency of blade ( $n=75 \mathrm{r} / \mathrm{min}$ ), due to the coupling effect between them, the revolution speed 
and phase angle variation of loading source also fluctuated significantly. Above test measure results were also basically match with the simulation results.
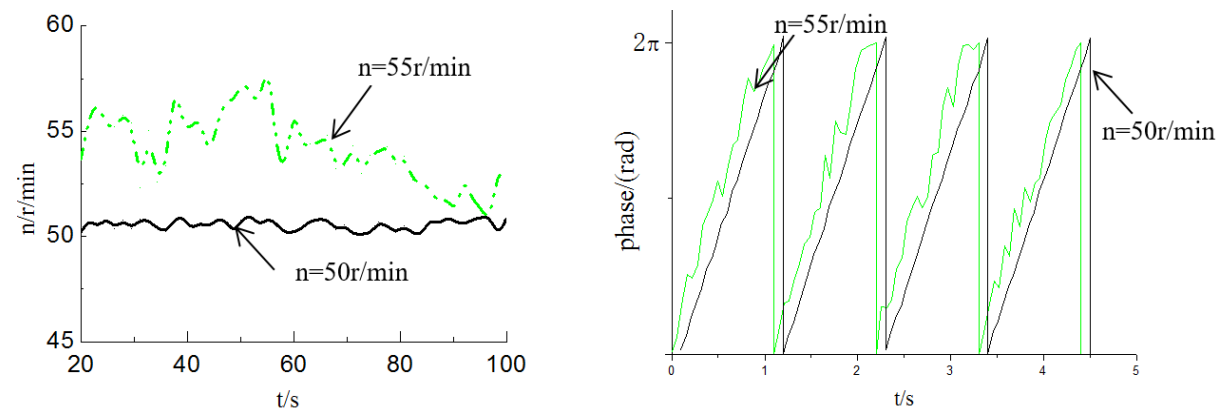

Fig. 12. Revolution speed and phase angle test curve of loading source in flapwise direction
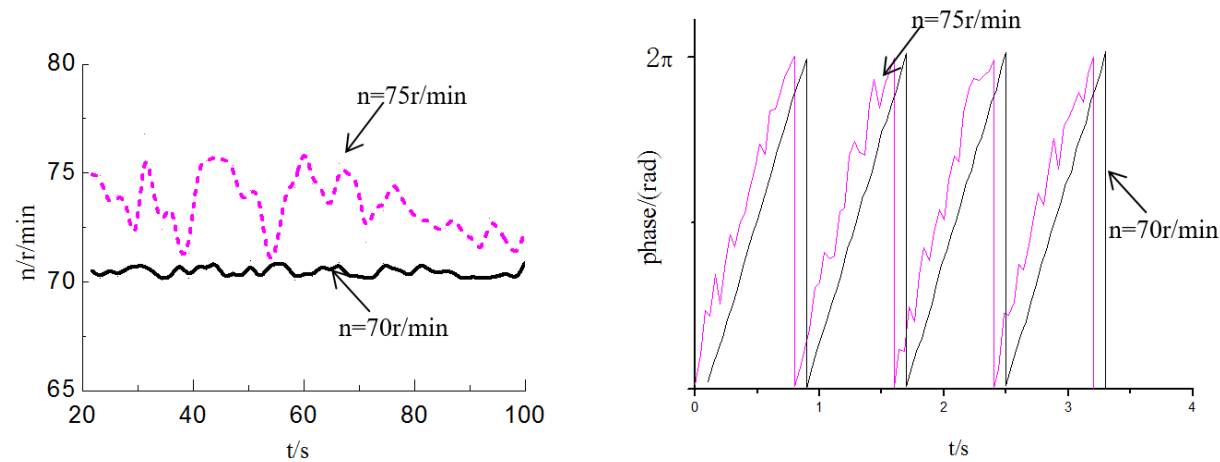

Fig. 13. Revolution speed and phase angle test curve of loading source in edgewise direction

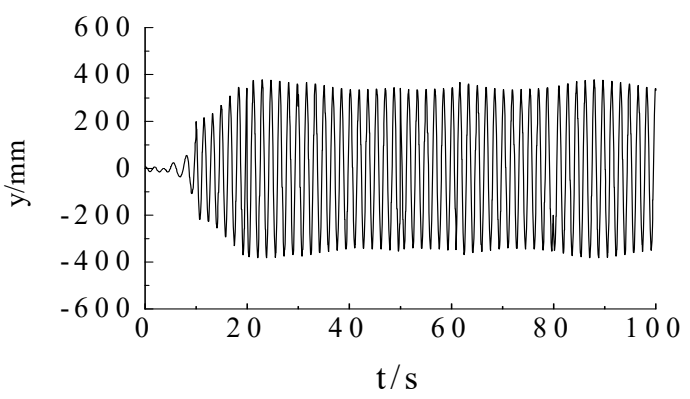

a) Amplitude test curve of flapwise direction in resonance

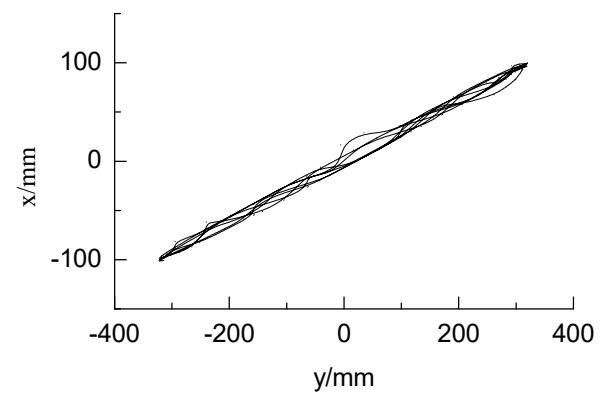

c) Vibration trajectory in resonance condition

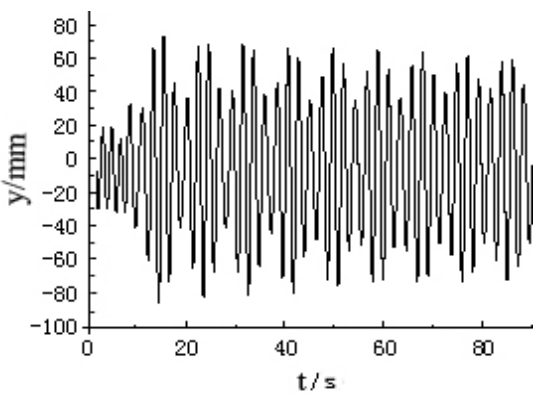

b) Amplitude test curve of flapwise

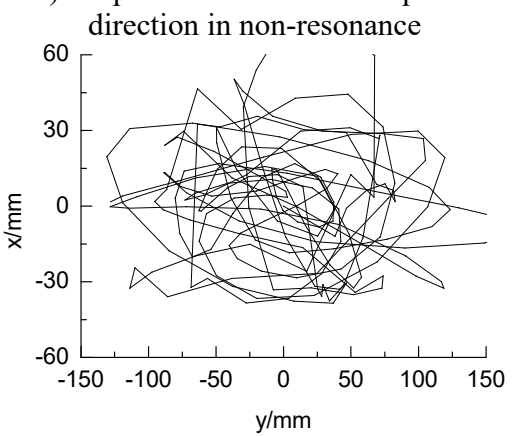

d) Vibration trajectory in non-resonance condition

Fig. 14. Vibration trajectory test curve of wind turbine blade 
When the revolution speed of loading source matched with the natural frequency of wind turbine blade, amplitude of blade was not only stable, but also maximum, about $400 \mathrm{~mm}$. The vibration trajectory was stable. Such condition was suitable for multi-axis fatigue loading test. When the setting revolution speed of the loading source was different with the natural frequency of the blade, the amplitude and vibration trajectory of the blade were in disorder because of the electromechanical coupling effect, as shown in Fig. 14.

\section{Conclusions}

In this paper, a new multi-axis fatigue loading system of wind turbine blade driven by electric mass was designed. The measurement method for revolution speed, phase angle of loading source and blade amplitude was given. The conclusions were obtained in follows.

1) In order to shorten the measuring time for wind turbine blade fatigue loading test, the multi-axis fatigue loading system based on electric mass driven was designed. By establishing the mathematical model for multi-axis fatigue loading system according to the Lagrange equation, it was found that the proposed system was an electromechanical coupling system. Its numerical solution was very difficult to get.

2) The vibration characteristics of multi-axis fatigue loading system were visually obtained through the numerical simulation model. When the revolution speeds of two loading source were same to the natural frequency of the blade vibration direction, the revolution speed and phase angle variation of loading source were basically stable. Moreover, the vibration trajectory was relatively smooth. The amplitude of blade was maximum, which was suitable for fatigue loading test. When the revolution speeds of loading source were different with natural frequency of wind turbine blade, the revolution speed and phase angle of loading source fluctuated significantly and vibration trajectory was rough. In order to obtain the better measurement effect, the corresponding decoupling control algorithm was needed in future.

3) A multi-axis fatigue loading experiment platform was developed. Using this test platform, the test result was basically same with the simulation result, which verified the accuracy of the mathematical model and simulation model in this paper. All the above findings have theoretical and engineering significance for the structure redesign of wind turbine blade

\section{Acknowledgement}

This work was sponsored by National Natural Science Foundation of China (No. 51405275 and No. 51305243) and Shandong Provincial Natural Science Foundation (No. ZR2014EL027) and China Postdoctoral Science Foundation (2015M571840).

\section{References}

[1] Liu Deshun, Dai Juchuan, Hu Yanping, et al. Status and development trends of modern large-scale wind turbines. China Mechanical Engineering, Vol. 24, Issue 1, 2013, p. 125-135.

[2] Kong C., Bang J., Sugiyama Y. Structural investigation of composite wind turbine blade considering various load cases and fatigue life. Energy, Vol. 30, 2005, p. 2101-2114.

[3] Huang Xuemei, Zhang Leian, Yuan Guangming, et al. Accuracy improvement on fatigue test of megawatt wind turbine blades by adaptive fuzzy control. Journal of Vibroengineering, Vol. 16, Issue 6, 2014, p. 2631-2638.

[4] White D., Musial W., Engberg S. Evaluation of the new B-REX fatigue testing system for multi-megawatt wind turbine blades. 43rd AIAA Aerospace Sciences Meeting and Exhibit, USA, Vol. 6, 2005, p. 10-13.

[5] Massicotte D., Megner B. Neural-network based method of correction in a nonlinear dynamic measuring system. IEEE Transaction on Instrumentation and Measurement, Vol. 39, Issue 4, 1999, p. 1641-1645.

[6] Suykens J. A. K. Nonlinear modeling and support vector machines. IEEE Instrumentation and Measurement Technology Conference, Budapest, 2001, p. 287-294.

[7] Xiao Chen, Wei Zhao, Xiao Lu Zhao, et al. Failure test and finite element simulation of a large wind turbine composite blade under static loading. Energy, Vol. 7, 2014, p. 2274-2297. 
[8] Wang Degang, Yao Hongliang, Feng Fei, et al. Dynamic coupling feature and experimental study of self-synchronous vibrating system. Journal of Vibration, Measurement Diagnosis, Vol. 32, Issue S2, 2012, p. 53-58.

[9] Zhang Tianxia, Xiaoyu E., Wen Bangchun Coupling effect in a synchronous vibration system. Journal of Northeastern University, Vol. 24, Issue 9, 2003, p. 839-842.

[10] Zhang Nan, Hou Xiaolin, Wen Bangchun Synchronous theory for multi-type self-synchronous vibration systems. China Mechanical Engineering, Vol. 30, Issue 15, 2009, p. 1838-1844.

[11] Lai Xin, Wu Jianzhong, Zhou Wen, et al. Synchronization control and electromechanical coupling characteristics of pile hammer synchronous vibration system. Journal of Tongji University, Vol. 48, Issue 6, 2012, p. 920-925.

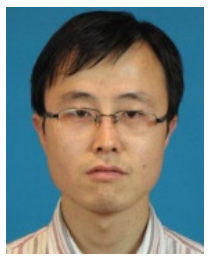

Zhang Lei'an received the B.S. degree in Mechanical Engineering from Weifang University, China, in 2005, and his M.S. and Ph.D. degrees in Mechanical Engineering from Shandong University of Technology and Tongji University, China, in 2008 and 2012, respectively. He is a lecturer of College of Mechanical Engineering, Shandong University of Technology. His research is about the performance testing of wind turbine blade.

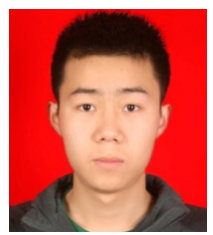

Tao Liming received the B.S. degree in mechanical engineering from Lu dong University, China, in 2015 and he was given the title of 3D CAD application engineer in 2013. He was a postgraduate student in Shandong University of Technology since 2015. His research is mainly about technique of mechanics and electronics and hydraulics integration.

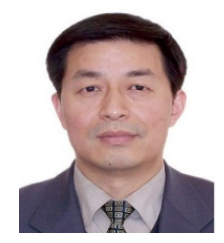

Wei Xiuting received his Bachelor degree in hydraulic machinery from Jiangsu University, China in 1982 and his $\mathrm{PhD}$ in machine design and theory from Zhejiang University, China in 1999. He is a Professor of mechanical engineering at Shandong University of Technology China. His research is mainly about electromechanical control.

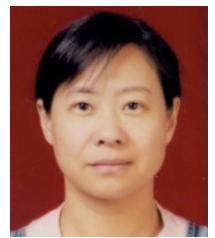

Huang Xuemei received the B.S. degree in Mechanical Engineering from Shandong University of Technology, China, in 1997, and her M.S. and Ph.D. degrees in Mechanical Engineering from Shandong University and Shanghai Jiaotong University, China, in 2001 and 2004, respectively. She is an Associate Professor in College of Mechanical Engineering, Shandong University of Technology. Her research interests include modeling and simulation of electromechanical system, electromechanical couple. 